\title{
Influence of viscous dissipation on the exiting sheet thickness in the calendering of Newtonian fluids
}

\author{
José C. Arcos \\ ESIME UA \\ Instituto Politécnico Nacional \\ México D.F., 02250 \\ Email: http://www.ipn.mx
}

\author{
Oscar E. Bautista \\ ESIME UA \\ Instituto Politécnico Nacional \\ México D.F., 02250 \\ Email:obautista@ipn.mx
}

\author{
Federico Méndez \\ Facultad de Ingeniería \\ UNAM \\ México D.F., 04510 \\ Email:fmendez@unam.mx
}

\author{
Juan P. Escandón \\ ESIME UA \\ Instituto Politécnico Nacional \\ México D.F., 02250 \\ Email:jescandon@ipn.mx
}

\begin{abstract}
In this work we treat theoretically the calendering process of Newtonian fluids with finite sheet initial thickness, taking into account that the viscosity of the fluid is a welldefined function of the temperature. We predict the influence of the temperature-dependent viscosity on the exiting sheet thickness in the calendering process. The mass, momentum and energy balance equations, based on the lubrication theory, were nondimensionalized and solved for the velocity, pressure and temperature fields by using perturbation and numerical techniques, where the exiting sheet thickness represents an eigenvalue of the mathematical problem. The numerical results show that the inclusion of temperature-dependent viscosity effect reduces about $20 \%$ the leave-off distance in comparison with the case of temperature-independent viscosity.
\end{abstract}

\section{INTRODUCTION}

The specialized literature for the calendering process is reviewed in the following paragraphs. The theoretical analysis regarding the above mechanism was developed by Gaskell [1] and McKelvey [2] for isothermal Newtonian fluid. Zheng and Tanner [?] applied the Phan-Thien-Tanner fluid model for the calendering process of inelastic and viscoelastic sheets using a perturbation method. Mitsoulis [3] numerically investigated the shape of the free surfaces of the entering and exiting sheets for the process of calendering viscoplastic sheets with a finite thickness. The combined effects of asymmetry and viscous heating for the non-isothermal nip flow in calendering were considered by Dobbels and Mewis [4]. The effect of viscous dissipation on calendering process of Newtonian and powerlaw fluids have been studied by Kiparissides and Vlachopoulos [5]. They reported the temperature profiles due to viscous dissipation in calendering gap, finding two maxima in the vicinity of the roll surfaces. In addition, the maximum temperatures exhibit two local maxima and one minimum in the direction of the flow. It appears that the only work about non-isothermal calendering of Newtonian fluids is just the aforementioned work. However, in this work they determined the temperature profile, disregarding the influence of the temperature on the final exiting sheet thickness. In this direction, Middleman [6] developed a simple model of the sensitivity of calendered thickness to temperature fluctuations. He concluded that " $a$ $3^{\circ}$ variation in temperature will cause more than 20 percent variation in calendered thickness!". In this sense, to our knowledge there are not works that validate the aforementioned sentence. Therefore, the goal of this work is to determine the influence of the viscous dissipation on the dimensionless

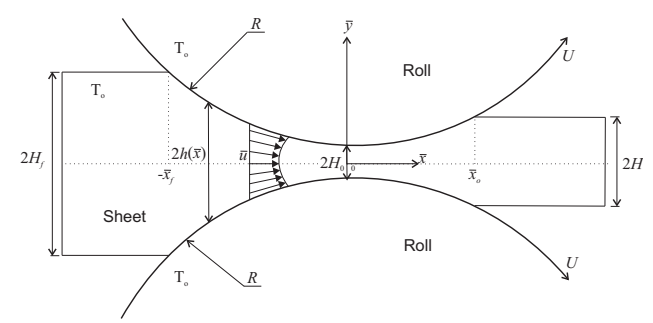

Fig. 1. Schematic diagram of the studied physical model, in physical variables.

exiting sheet thickness for the calendering process taking into account the temperature-dependent viscosity .

\section{A. Formulation}

In Fig. 1, we show a sketch of the studied physical model. Two cylinders separated by a thin film of a Newtonian liquid with temperature dependent viscosity, are rotated in the same direction. Each cylinder has a radius $R$, rotating with a constant angular velocity, $\omega$, resulting in a linear velocity at its surface given by $U=\omega R$. The minimum gap half-height, $H_{0}$, is such that $H_{0} \ll R$ and the one-half exiting sheet thickness is represented by $H$. The geometry of the roll surface is given as $h(\bar{x})=H_{0}\left(1+\bar{x}^{2} / 2 R H_{0}\right)[6]$. We assume that the roll surfaces are found at a constant temperature, $T_{0}$. The location $\bar{x}$ where the sheet first bites the rolls is represented by $-\bar{x}_{f}$, which is known. On the other hand, the leave-off distance location of the sheet, modified by temperature effects, represented by $\lambda$ is unknown, and must be determined in the present analysis. The mass, momentum and energy equations, in steady state, are the following

$$
\begin{gathered}
\frac{\partial \bar{u}}{\partial \bar{x}}+\frac{\partial \bar{v}}{\partial \bar{y}}=0 \\
\rho \bar{u} \frac{\partial \bar{u}}{\partial \bar{x}}+\rho \bar{v} \frac{\partial \bar{u}}{\partial \bar{y}}=-\frac{\partial \bar{P}}{\partial \bar{x}}+\frac{\partial \bar{\tau} y x}{\partial \bar{y}} \\
\rho \bar{u} \frac{\partial \bar{v}}{\partial \bar{x}}+\rho \bar{v} \frac{\partial \bar{v}}{\partial \bar{y}}=-\frac{\partial \bar{P}}{\partial \bar{y}}+\frac{\partial \bar{\tau}_{x y}}{\partial \bar{x}} \\
\rho c_{p} \bar{u} \frac{\partial T}{\partial \bar{x}}=k\left(\frac{\partial^{2} T}{\partial \bar{x}^{2}}+\frac{\partial^{2} T}{\partial \bar{y}^{2}}\right)+\bar{\tau}_{x y} \frac{\partial \bar{u}}{\partial \bar{y}}
\end{gathered}
$$




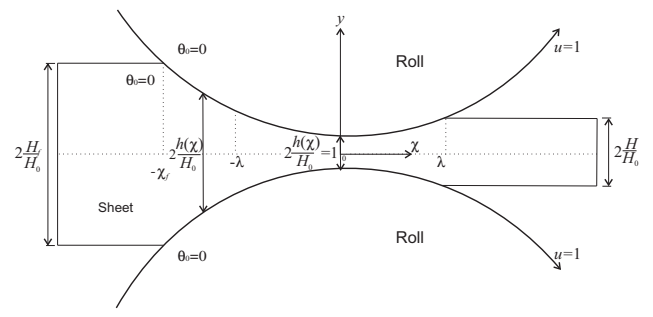

Fig. 2. Schematic diagram of the studied physical model, in dimensionless variables.

where the shear stress is given as [7],

$$
\bar{\tau}_{x y}=\bar{\tau}_{x y}=\mu_{0} \exp \left[-a\left(T-T_{0}\right)\right]\left(\frac{\partial \bar{u}}{\partial \bar{y}}+\frac{\partial \bar{v}}{\partial \bar{x}}\right) .
$$

The boundary and initial conditions associated with Eqs. (1)-(4) are

$$
\begin{gathered}
\bar{y}=0: \frac{\partial \bar{u}}{\partial \bar{y}}=0, \\
\bar{y}=h(\bar{x}): \bar{u}=U, \\
\bar{y}=0: \frac{\partial T}{\partial \bar{y}}=0, \\
\bar{y}=h(\bar{x}): T=T_{0}, \\
\bar{x}=-\bar{x}_{f}: T=T_{0},
\end{gathered}
$$

In the region of the nip and extending to either side (i. e., in the $\pm \bar{x}$ direction) by a distance of the order of $\bar{x}_{0}$ (see Fig. 1), the roll surface are nearly parallel when $H_{0} \ll R$. Then, we can assume that the flow is nearly parallel, so that $\bar{v} \ll \bar{u}$ and $\frac{\partial}{\partial \bar{x}} \ll \frac{\partial}{\partial \bar{y}}$, as we will show below. According to this assumption, the boundary conditions (6-10) are those used in the lubrication approximation theory.

In the above equations, $\bar{u}, \bar{v}, \bar{P}$ and $T$ represent the velocity components in $\bar{x}$ and $\bar{y}$ directions, pressure and temperature fields of the fluid, respectively. $\rho, c_{p}, k, \mu_{0}$ are the density, specific heat, thermal conductivity and the viscosity of the Newtonian liquid evaluated at a reference temperature $T_{0}$, respectively. In addition, $a$ is an empirical parameter that measures the dependence of the viscosity on the temperature; this constant is of order of $10^{-2}$ to $10^{-1}[6,7]$. In Eq. (5) we have included the exponential temperature dependence model for the viscosity [7].
1) Dimensionless Equations: In this section, we present the dimensionless governing equations needed to solve the nonisothermal calendering process, Fig. 2. Based on the order of magnitude analysis carried out previously, we can define the following dimensionless variables

$$
\begin{aligned}
\chi & =\frac{\bar{x}}{\sqrt{2 R H_{0}}}, \quad y=\frac{\bar{y}}{H_{0}}, \quad \frac{h(\bar{x})}{H_{0}}=1+\chi^{2} \\
P & =\frac{\bar{P} H_{0}}{\mu_{0} U}, \quad \lambda^{2}=\frac{H}{H_{0}}-1, \quad Q(\chi)=\frac{\bar{Q}}{2 U H_{0}} \\
u(\chi, y) & =\frac{\bar{u}(\bar{x}, \bar{y})}{U}, v(\chi, y)=\frac{\bar{v}(\bar{x}, \bar{y}) \sqrt{2 R H_{0}}}{U H_{0}} \\
\theta(\chi, y) & =\sqrt{\frac{H_{0}}{2 R}} \frac{\left(T(\bar{x}, \bar{y})-T_{0}\right) \rho_{f} c_{p} H_{0}}{\mu_{0} U} .
\end{aligned}
$$

Introducing the dimensionless variables defined by previous relationships (11) into the conservation equations (1)-(5), we obtain

$$
\begin{gathered}
\operatorname{Re} \beta\left(u \frac{\partial u}{\partial \chi}+v \frac{\partial u}{\partial y}\right)=-\beta \frac{d P}{d \chi}+\frac{\partial}{\partial y}\left(e^{-\epsilon \theta} \frac{\partial u}{\partial y}\right), \\
G z u \frac{\partial \theta}{\partial \chi}=\frac{\partial^{2} \theta}{\partial y^{2}}+G z e^{-\epsilon \theta}\left(\frac{\partial u}{\partial y}\right)^{2} .
\end{gathered}
$$

Here, $\epsilon$ is a dimensionless parameter, given as $\epsilon=N a / G z$, where $N a$ is the Nahme-Griffith number $[8,9]$, defined as $N a=a\left(\mu_{0} U^{2} / k\right)$, and $G z$ is the well-known Graetz number, given by $G z=\beta P e$. Here $P e$ is the Peclet number, represented by $P e=\left(\rho c_{p} / k\right) U H_{0}$, and $\beta$ is a geometric parameter, defined as $\beta=\left(H_{0} / 2 R\right)^{1 / 2}$. The Nahme-Griffith number represents the ratio of the temperature rise, due to viscous dissipation to the temperature rise needed to change the viscosity. $R e$ is the Reynolds number, defined as $R e=\rho U H_{0} / \mu_{0}$. In typical applications of calendering, $\epsilon \ll 1$, in such a way that the exponential factor, in the momentum and energy equations, can be linearized as $e^{-\epsilon \theta} \approx 1-\epsilon \theta+\cdots$, and the Reynolds number is much less than unity, $R e \sim 10^{-4}-10^{-5}$, indicating that the inertia plays a minor role in this process; the Peclet number takes values of order of $10^{2}$ to $10^{3}$. According to the previous discussion, Eq. (12) transforms to

$$
\beta \frac{d P}{d \chi}=\frac{\partial}{\partial y}\left[(1-\epsilon \theta+\cdots) \frac{\partial u}{\partial y}\right],
$$

with the following dimensionless boundary conditions,

$$
\begin{gathered}
\left.\frac{\partial u}{\partial y}\right|_{y=0}=0, \\
u\left(y=1+\chi^{2}\right)=1 .
\end{gathered}
$$

Furthermore, Eq. (14) requires additional boundary conditions for the pressure gradient $d P / d \chi$ and the pressure $P$, given by

$$
\begin{gathered}
\left.\frac{d P}{d \chi}\right|_{\chi=\lambda}=P(\chi=\lambda)=0, \\
P\left(\chi=-\chi_{f}\right)=0 .
\end{gathered}
$$


On the other hand, the energy equation, Eq. (13), takes the form

$$
G z u \frac{\partial \theta}{\partial \chi}=\frac{\partial^{2} \theta}{\partial y^{2}}+G z(1-\epsilon \theta+\cdots)\left(\frac{\partial u}{\partial y}\right)^{2},
$$

with the boundary conditions,

$$
\begin{gathered}
\theta\left(-\chi_{f}, y\right)=0 \\
\theta\left(\chi, 1+\chi^{2}\right)=0 \\
\left.\frac{\partial \theta}{\partial y}\right|_{y=0}=0 .
\end{gathered}
$$

Together with Eqs. (14) and (19), we need the dimensionless mass balance equation, which can be written in the form,

$$
Q=1+\lambda^{2}=\int_{0}^{1+\chi^{2}} u d y,
$$

where $Q$ is the volumetric flow rate and for the present formulation, assumes a constant value. In addition, $\lambda$ represents an unknown eigenvalue of the mathematical problem and is related to the exiting sheet thickness for the calendering process through the following relationship $\lambda^{2}=H / H_{0}-1$. We anticipate that this parameter is directly influenced by temperature effects in contrast to those that do not consider the inclusion of these effects. The system of equations (14) and (19) represent the well known lubrication approximation [8] for a Newtonian liquid with temperature-dependent viscosity.

From the classical calendering analysis $[1,8]$, for Newtonian liquids, there are two flow regions in the $x$-direction: one region near to the entrance and other at the exit of the nip. These two regions have a positive pressure gradient in the domain $-\chi_{f} \leq \chi \leq-\lambda$ and a negative pressure gradient, in this other interval $-\lambda \leq \chi \leq \lambda$. In the following section and for each region, we obtain the corresponding velocity, pressure and temperature profiles.

\section{B. Asymptotic solution for the limit $\epsilon \ll 1$.}

To determine the dimensionless velocity, pressure, temperature profiles, leave-off distance and the corresponding exiting thickness of the calendered material, we conduct an asymptotic solution by decoupling the system of Eqs. (14) and (19). Applying a regular perturbation technique and using $\epsilon$ as the perturbation parameter, we propose the following expansions,

$$
\begin{gathered}
u(\chi, y)=u_{0}(\chi, y)+\epsilon u_{1}(\chi, y)+\cdots \\
P(\chi)=P_{0}(\chi)+\epsilon P_{1}(\chi)+\cdots \\
Q=Q_{0}+\epsilon Q_{1}+\cdots
\end{gathered}
$$

$$
\begin{gathered}
\theta(\chi, y)=\theta_{0}(\chi, y)+\epsilon \theta_{1}(\chi, y)+\cdots, \\
\lambda=\lambda_{0}+\epsilon \lambda_{1}+\cdots,
\end{gathered}
$$

where $u_{0}, P_{0}, Q_{0}, \lambda_{0}$ and $\theta_{0}$ are the leading-order solutions for the isothermal case and solved in previous works $[1,2,8]$. $P_{1}, Q_{1}, u_{1}, \theta_{1}$ and $\lambda_{1}$ are corrections up to terms of firstorder. Introducing the relationships (24)-(28) into Eqs. (14)(23), we obtain, after collecting terms of the same power of $\epsilon$, the following sets of equations

for $\epsilon^{0}$ :

$$
\begin{gathered}
\beta \frac{d P_{0}}{d \chi}=\frac{\partial^{2} u_{0}}{\partial y^{2}}, \quad \text { for } \quad-\chi_{f} \leq \chi \leq \lambda_{0}, \\
Q_{0}=1+\lambda_{0}^{2}=\int_{0}^{1+\chi^{2}} u_{0} d y, \\
G z u_{0} \frac{\partial \theta_{0}}{\partial \chi}=\frac{\partial^{2} \theta_{0}}{\partial y^{2}}+G z\left(\frac{\partial u_{0}}{\partial y}\right)^{2} .
\end{gathered}
$$

The boundary conditions for Eqs. (29)-(31) are:

$$
\begin{gathered}
\frac{\partial u_{0}}{\partial y}=0 \text { and } \frac{\partial \theta_{0}}{\partial y}=0, \quad \text { at } y=0, \\
u_{0}=1 \text { and } \theta_{0}=0, \quad \text { at } y=1+\chi^{2}, \\
P_{0}=0 \text { and } \theta_{0}=0, \text { at } \chi=-\chi_{f}, \\
\frac{d P_{0}}{d \chi}=P_{0}=0 \text { at } \chi=\lambda_{0} .
\end{gathered}
$$

For the first order solution, $\epsilon^{1}$ :

$$
\begin{gathered}
\beta \frac{d P_{1}}{d \chi} y=\frac{\partial u_{1}}{\partial y}-\theta_{0} \frac{\partial u_{0}}{\partial y}, \text { for }-\chi_{f} \leq \chi \leq \lambda_{1} \\
Q_{1}=2 \lambda_{0} \lambda_{1}=\int_{0}^{1+\chi^{2}} u_{1} d y
\end{gathered}
$$

For solving Eqs. (36) and (37), we need the zeroth-order solution for mass, momentum and energy equations, Eqs. (29)(31). The boundary conditions for Eqs. (36) and (37) are written as,

$$
\begin{gathered}
\frac{\partial u_{1}}{\partial y}=0 \text { and } \frac{\partial \theta_{1}}{\partial y}=0, \quad \text { at } y=0, \\
u_{1}=0 \text { and } \theta_{1}=0, \text { at } y=1+\chi^{2}, \\
P_{1}=0 \text { and } \theta_{1}=0, \quad \text { at } \quad \chi=-\chi_{f},
\end{gathered}
$$




$$
\frac{d P_{1}}{d \chi}=P_{1}=0 \quad \text { at } \quad \chi=\lambda_{1}
$$

1) Zeroth-order solution: The solution of Eq.(29), considering a finite feed thickness, is given by:

$$
u_{0}=1+\frac{1}{2} \beta\left(\frac{d P_{0}}{d \chi}\right)\left[y^{2}-\left(1+\chi^{2}\right)^{2}\right]
$$

with

$$
\frac{d P_{0}}{d \chi}=-3 \beta^{-1} \frac{\left(\lambda_{0}^{2}-\chi^{2}\right)}{\left(1+\chi^{2}\right)^{3}}
$$

for $-\chi_{f} \leq \chi \leq \lambda_{0}$.

We must emphasize that the zeroth-order solution, Eqs. (42) and (43), were obtained in previous works $[1,8]$. In the present analysis, the zeroth-order energy equation, Eq. (31), was solved in the two flow regions in the $\chi$ direction: one region near to the nip at the entrance to the rolls, which has a positive pressure gradient; and the other region away from the nip at the exit, with opposite sign for the pressure gradient.

Due to the curvature of the rolls, we use a simple independent variable transformation that makes possible to solve the dimensionless energy equation on a uniformly spaced computational grid, therefore we can introduce the following transformation variable, $Y=y /\left(1+\chi^{2}\right)$, then Eq. (31) takes the form,

$$
G z u_{0} \frac{\partial \theta_{0}}{\partial \chi}=\frac{1}{\left(1+\chi^{2}\right)^{2}} \frac{\partial^{2} \theta_{0}}{\partial Y^{2}}+G z\left(\frac{1}{1+\chi^{2}} \frac{\partial u_{0}}{\partial Y}\right)^{2}
$$

In the new independent variable, $Y$, the zeroth-order velocity profile is given by

$$
u_{0}=1+\frac{3}{2} \frac{\left(\chi^{2}-\lambda_{0}^{2}\right)}{1+\chi^{2}}\left(Y^{2}-1\right)
$$

the boundary conditions, given by Eqs. (32) and (33), associated with the Eq. (44) are transformed to

$$
\begin{gathered}
Y=0: \frac{\partial \theta_{0}}{\partial Y}=0 \\
Y=1: \theta_{0}=0
\end{gathered}
$$

Equation (44) was numerically solved by using the conventional Crank-Nicolson method. To solve this equation, we selected a mesh with 501 nodal points for the half width and 1027 in the direction of the flow. The details are omitted for simplicity.

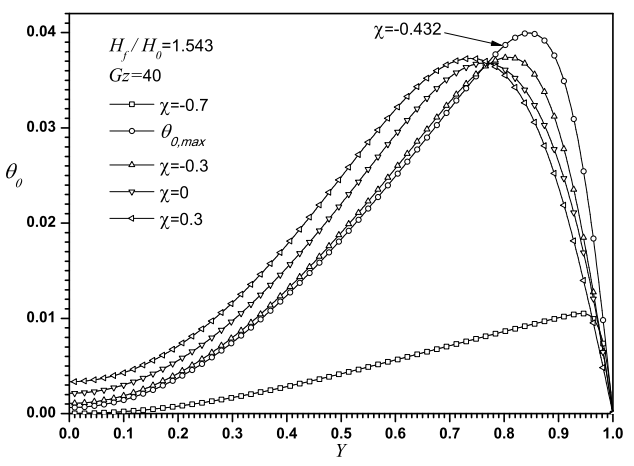

Fig. 3. Dimensionless temperature $\theta_{0}$ as a function of the dimensionless longitudinal $\chi$ and transversal $Y$ coordinates, $G z=40, H_{f} / H_{0}=1.543$, at five different positions of the variable $\chi$

2) First order solution: The solution procedure for the firstorder is similar to the zeroth-order solution; we begin integrating Eq. (36) to find $u_{1}$ explicitly as a function of $Y$, In terms of the independent variable transformation $y=\left(1+\chi^{2}\right) Y$, after applying the boundary conditions (38) and (39) takes the form

$$
\begin{array}{r}
u_{1}(\chi, Y)=\frac{1}{2} \beta\left(1+\chi^{2}\right)^{2} \frac{d P_{1}}{d \chi}\left[Y^{2}-1\right] \\
-\beta\left(1+\chi^{2}\right)^{2} \frac{d P_{0}}{d \chi}\left[\int_{0}^{1} \theta_{0} Y d Y-\int_{0}^{Y} \theta_{0} Y d Y\right]
\end{array}
$$

Replacing Eq. (48) into Eq. (37), and applying the boundary condition, given by Eq. (41) we obtained the volumetric flow rate for the first order and after some algebraic manipulations, we can also obtain one explicit expression for $d P_{1} / d \chi$

$$
\begin{gathered}
Q_{1}=\frac{\left(-\frac{d P_{0}}{d \chi}\right)_{\chi=\lambda_{1}}}{\left(\beta^{-1}\left(1+\lambda_{1}^{2}\right)^{-3}\right)}\left\{\begin{array}{c}
{\left[\int_{0}^{1} \int_{0}^{1} \theta_{0} Y d Y d Y\right.} \\
\left.-\int_{0}^{1} \int_{0}^{Y} \theta_{0} Y d Y d Y\right]
\end{array}\right\} \\
\frac{d P_{1}}{d \chi}=\frac{9}{\beta}\left\{\begin{array}{c}
\frac{\left(\lambda_{0}^{2}-\chi^{2}\right)}{\left(1+\chi^{2}\right)^{3}}\left[\int_{0}^{1} \int_{0}^{1} \theta_{0} Y d Y d Y\right. \\
\left.-\int_{0}^{1} \int_{0}^{Y} \theta_{0} Y d Y d Y\right] \\
-\frac{\left(\lambda_{0}^{2}-\lambda_{1}^{2}\right)}{\left(1+\chi^{2}\right)^{3}}\left[\int_{0}^{1} \int_{0}^{1} \theta_{0} Y d Y d Y\right. \\
\left.-\int_{0}^{1} \int_{0}^{Y} \theta_{0} Y d Y d Y\right]_{\chi=\lambda_{1}}
\end{array}\right\}
\end{gathered}
$$

2) This equation is valid for $-\chi_{f} \leq \chi \leq \lambda_{1}$, where $\lambda_{1}$ must be determined as a part of the problem. In the above equation, the procedure to evaluate the integral terms is the following: at each axial position , $\chi$, the term given by the first integral, $\int_{0}^{1} \int_{0}^{1} \theta_{0} Y d Y d Y$, is found numerically by using the Trapezoidal rule. Specifically, this procedure was implemented at each axial position, $\chi$, from the point where the entering sheet first bites the rolls [8] to the leave-off distance point of the rolls, given by the zeroth order solution of $\lambda_{0}$. For the obtained data in this numerical integration, one ninth-degree 


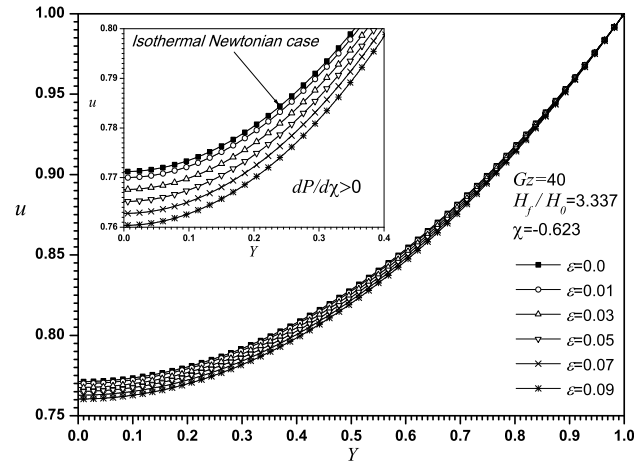

Fig. 4. Dimensionless velocity distribution $u$ for different values of the parameter $\varepsilon$, as a function of the dimensionless transversal coordinate $Y$, evaluated at $\chi=-0.623$, for $H_{f} / H_{0}=3.337$ and $G z=40$.

polynomial regression was generated as a function of the axial coordinate $\chi$.

For the second integral, $\int_{0}^{1} \int_{0}^{Y} \theta_{0} Y d Y d Y$, at each axial position, $\chi$, we have obtained the data points, $\left(Y_{j}, \theta_{o, j} Y_{j}\right)$, where $j$ represents the position for each transverse location and from which we can fit a nineth-degree polynomial for the $Y$ coordinate. Once this is done, each resulting polynomial was integrated from $Y=0$ to $Y=1$ using the Trapezoid rule. We omit the presentation of these polynomials because approximately 1000 polynomials were generated.

3) In order to find the first order of the dimensionless flow rate, $Q_{1}$, and consequently $\lambda_{1}$, an iterative procedure was applied to Eq. (50). At the starting of the iterative procedure, we assume a value for $\lambda_{1}$. Once this is done, the first order of dimensionless pressure $P_{1}(\chi)$ was obtained by integrating Eq. (50) using a conventional fourth-order Runge-Kutta technique. The procedure was repeated until the boundary condition given by Eq. (41) was fully satisfied.

\section{RESULTS}

For the numerical results presented in this section, we have used representative values of the parameters involved in the calendering process $[9,10]$. Figure 3 shows the numerical solution for the dimensionless temperature $\theta_{0}$ as a function of the dimensionless transverse direction at five axial positions, $\chi$, for two fixed different entering sheet thickness, $H_{f} / H_{0}(=1.543$, 3.337 ) and $G z=40$, respectively. In these figures, we can note that the maximum values of the dimensionless temperature are confined to the vicinity of the roll surface for each $\chi$ position. These are the results of the interaction between the viscous dissipation of the fluid and the heat transfer mechanisms to the rolls. For the same figures, the larger values of the dimensionless temperature in the $\chi$ direction are located near to the entry.

In Figs. 4 and 5, the velocity profiles were evaluated in a position $\chi$, which corresponds to the region where pressure gradient has a positive value, and the other region with opossite sign. From these figures, we can see that the dimensionless velocity $u$ decreases as the parameter $\epsilon$ increases. This decrease on the dimensionless velocity is insignificant in the vicinity of the rolls and more pronounced at the center plane.

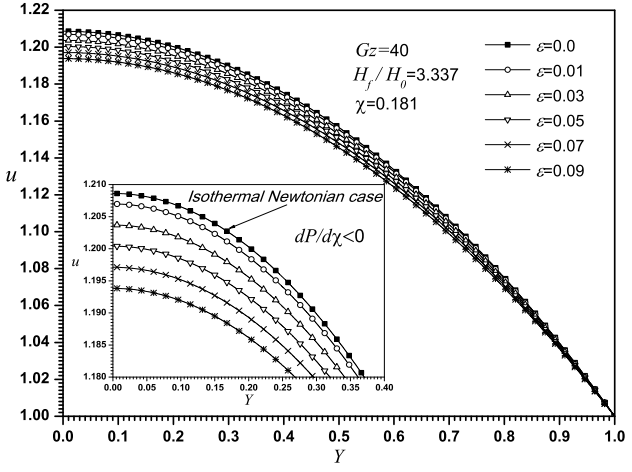

Fig. 5. Dimensionless velocity distribution $u$ for different values of the parameter $\varepsilon$, as a function of the dimensionless transversal coordinate $Y$, evaluated at $\chi=0.181$, for $H_{f} / H_{0}=3.337$ and $G z=40$.

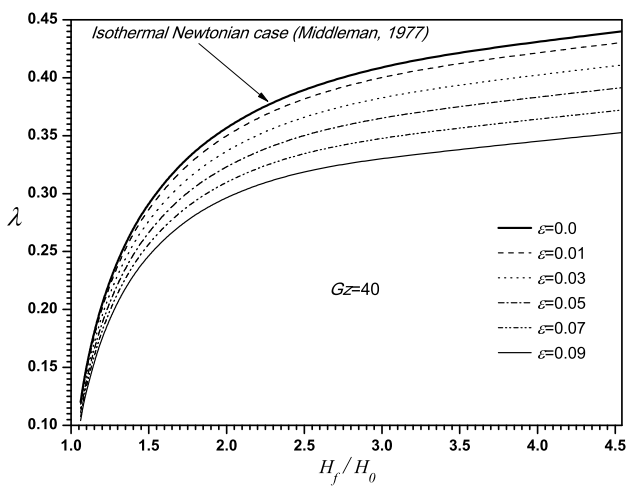

Fig. 6. The dimensionless leave-off distance $\lambda$ as a function of the entering sheet thickness $H_{f} / H_{0}$ for different values of the parameter $\epsilon$, for a Newtonian fluid with $G z=40$.

In Fig. 6 we show the complete result for the dimensionless leave-off distance, $\lambda$, as a function of the dimensionless entering sheet thickness, $H_{f} / H_{0}$, under the influence of the parameters $\epsilon$, for $G z=40$. In order to determine the correction $\lambda_{1}$ due to thermal effects, Eq. (50) was solved iteratively as a function of $\lambda_{0}, \theta_{0}$ and $u_{0}$. The obtained results for $\lambda_{0}$ and $\lambda_{1}$ are replaced in the expansion of $\lambda$, given by $\lambda=\lambda_{0}+\epsilon \lambda_{1}+\cdots$, for different values of the parameter $\epsilon$. In this work, we have assumed that the rolls were fed with a sheet of fluid with finite thickness, as suggested in Fig. 1. In this case, the dimensionless entering sheet thickness $H_{f} / H_{0}$ is a known parameter as it is shown in Fig. 2. According the above comments, the dimensionless entry distance, $-\chi_{f}$, is calculated following the relationship $\chi_{f}=\left(H_{f} / H_{0}-1\right)^{1 / 2}$. In all these figures, we can see that $\lambda$ and $H / H_{0}$ increase for increasing values of $H_{f} / H_{0}$, however $\lambda$ and $H / H_{0}$ decrease for increasing values of $\epsilon$. By comparison, in these figures we have included the case when no thermal effects are included $(\epsilon=0)$, which corresponds to the solution obtained by Middleman [8]. In these figures, for a fixed value of $H_{f} / H_{0}$, the leave-off distance and the exiting sheet thickness decreases as the parameter $\epsilon$ increases. It is evident that non-isothermal Newtonian case produces thinner sheets than the isothermal Newtonian fluid. With the parametric values used in Figs. 9 and 10 , the prediction of $\lambda$ and $H / H_{0}$ show that these variables are strongly dependent on the assumed values of the parameter $\epsilon$. For $H_{f} / H_{0}=4.54$ and $\epsilon=0.09$, the leave-off distance 
was reduced in about $20 \%$, compared with the Newtonian isothermal case.

\section{CONCLUSIONS}

In this paper, we have studied theoretically the influence of the viscous dissipation and temperature-dependent consistency index on the exiting sheet thickness, for a Newtonian fluid flowing between two cylinders rotating at the same velocity and temperature. The solution is based on the regular perturbation technique and the resulting governing equations are based on the well-known Lubrication theory. The numerical results predict the dimensionless velocity field, pressure, temperature distribution, and the exit locations and the exiting sheet thickness corrected by thermal effects. A decrease of $20 \%$ in the in the leave-off distance was determined. As it has been shown, in the limit of $\epsilon \rightarrow 0$, the present solution smoothly approaches to the unperturbed or zeroth-order solution, corresponding just to the isothermal case previously reported by Middleman [8].

\section{ACKNOWLEDGMENT}

This work has been supported by the research grants no. 169849 of Consejo Nacional de Ciencia y Tecnología of Mexico and no. 20131535 of SIP-IPN.

\section{REFERENCES}

[1] R. E. Gaskell, The calendering of plastic materials, Journal of Applied Mechanics17, pp. 334-337.

[2] J. M. McKelvey, Polymer Processing, Wiley, New York,1962

[3] R. Zheng and R. I. Tanner, A numerical analysis of calendering, Journal of Non-Newtonian Fluid Mechanics28, pp. 149170.

[4] S. Sofou and E. Mitsoulis, Calendering of pseudoplastic and viscoplastic sheets of finite thickness, Journal of Plastic Film Sheeting20, July, pp. 185-222.

[5] E. Mitsoulis, Numerical simulation of calendering viscoplastic fluids, Journal of Non-Newtonian Fluid Mechanics154 March, pp. 77-88.

[6] F. Dobbels and J. Mewis, Nonisothermal nip flow in calendering operation, AIChE Journal23(3), May, pp. 224-232

[7] C. Kiparissides and J. Vlachopoulos, A study of viscous dissipation in the calendering of power-law fluids, Polymer Engineering and Science18(3), pp. 210-214.

[8] S. Middleman, Fundamentals of Polymer ProcessingMcGraw-Hill, United States of America

[9] Z. Tadmor and C. G. Gogos, Principles of Polymer ProcessingJohn Wiley Sons, Haifa, Israel

[10] J. A. Dantzig and C. L. Tucker, Modeling in Materials ProcessingAddison-Wesley, Cambridge University Press

\section{Creative Commons Attribution License 4.0 (Attribution 4.0 International, CC BY 4.0)}

This article is published under the terms of the Creative Commons Attribution License 4.0

https://creativecommons.org/licenses/by/4.0/deed.en US 